\title{
Field-Free Three-Dimensional Alignment of Polyatomic Molecules
}

\author{
Kevin F. Lee, ${ }^{1,2}$ D. M. Villeneuve, ${ }^{1}$ P. B. Corkum, ${ }^{1,2}$ Albert Stolow, ${ }^{1}$ and Jonathan G. Underwood ${ }^{3, *}$ \\ ${ }^{1}$ Steacie Institute for Molecular Sciences, National Research Council Canada, Ottawa, Ontario, K1A OR6, Canada \\ ${ }^{2}$ Department of Physics and Astronomy, McMaster University, 1280 Main Street West, Hamilton, Ontario, L8S 4M1, Canada \\ ${ }^{3}$ Department of Physics and Astronomy, The Open University, Walton Hall, Milton Keynes, MK7 6AA, United Kingdom
}

(Received 28 June 2006; published 26 October 2006)

\begin{abstract}
We experimentally demonstrate field-free, three-dimensional alignment (FF3DA) of polyatomic asymmetric top molecules. We achieve FF3DA in sulfur dioxide gas using two time-delayed, orthogonally polarized, nonresonant, femtosecond laser pulses. Our method avoids the use of rotational revivals and is therefore more robust to temperature. The alignment is probed using time-delayed coincidence Coulomb explosion imaging. FF3DA will be important for all molecular imaging, dynamics, or spectroscopy experiments for which random alignment leads to a loss of information.
\end{abstract}

DOI: 10.1103/PhysRevLett.97.173001

PACS numbers: 33.15.Bh, 33.55.Be, 33.80.-b

The random alignment of gas phase molecules generally reduces the information content of a measurement made in the laboratory frame. The situation is analogous to the well-known case of powder, as opposed to crystal, x-ray diffraction. To ameliorate this situation, it is necessary to define the direction of the molecules in the lab frame prior to making a measurement. Polyatomic molecules are generally asymmetric rotors with three distinct axes of rotation. Three-dimensional alignment, i.e., the alignment of all three molecular axes, was achieved in the presence of an aligning laser field [1,2], but this field strongly perturbs the system, distorting the electronic and vibrational structure of the molecule [3] and preventing the measurement of innate molecular properties. Field-free one-dimensional alignment, i.e., alignment of a single molecular axis, was achieved using a short laser pulse [4,5], and by the rapid turn off of an adiabatic strong laser field [6]. In these experiments, maximal alignment is produced after the pulse $[4,7]$ when the molecule is field-free, followed by periodic revivals of the rotational wave packet [4]. The rotational energy level spacings for asymmetric tops are much less regular than for linear and symmetric top molecules. This complicates the rotational wave-packet evolution for asymmetric rotors, reducing alignment at revivals. The degradation of alignment worsens at elevated rotational temperatures due to the incoherent contributions of thermally populated rotational states which can lead to complete obfuscation of the rotational revival structure. This is a challenge for experimentalists since rotational cooling is often compromised in order to produce sufficiently dense molecular beams.

Here we report the experimental demonstration of a general, flexible, and robust method for producing fieldfree, three-dimensional alignment (FF3DA) of polyatomic molecules. This method makes use of the prompt alignment occurring just after the laser pulse and is much more robust with respect to temperature effects than is an earlier proposal for FF3DA at rotational revivals [8]. This tech- nique is broadly applicable and we demonstrate it here for the asymmetric top molecule $\mathrm{SO}_{2}$.

Our method is based upon the use of two time-separated, perpendicularly polarized, nonresonant, femtosecond laser pulses. The first laser pulse produces postpulse 1D alignment of the most polarizable axis of the molecule, at which point the second pulse produces a torque about the most polarizable axis to bring the second most polarizable axis into alignment with the second laser field polarization direction, producing FF3DA after the second pulse. We illustrate this method in Fig. 1 for $\mathrm{SO}_{2}$. A randomly oriented ensemble of $\mathrm{SO}_{2}$ molecules is illustrated in the first frame of Fig. 1. Application of a linearly polarized nonresonant laser pulse (blue shading) exerts a torque on each molecule. Although the magnitude and direction of this torque depends on the molecular orientation, it tends to "kick" the most polarizable molecular axis towards alignment with the laser polarization. For the case of $\mathrm{SO}_{2}$, the most polarizable axis is along the $\mathrm{O}-\mathrm{O}$ direction (called hereafter the $\mathrm{O}$ axis). We choose this first pulse to be shorter than the time required for the molecule to rotate about the axes perpendicular to the $\mathrm{O}$ axis: maximal alignment of the $\mathrm{O}$ axis thus occurs after the laser pulse. As shown in the second frame of Fig. 1, although the $\mathrm{O}$ axis is

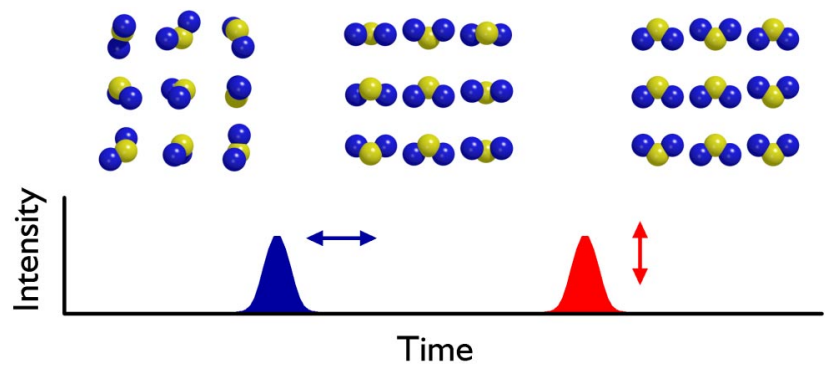

FIG. 1 (color online). Scheme for field-free 3D alignment of $\mathrm{SO}_{2}$ molecules using time-separated laser fields. $\mathrm{S}$ atoms are shown in yellow and $\mathrm{O}$ atoms are shown in blue. For details see text. 
confined to the laser polarization, there is free rotation of the molecules about the $\mathrm{O}$ axis. At this point, the alignment is $1 \mathrm{D}$ due to the cylindrically symmetric nature of the interaction: only the $\mathrm{O}$ axis direction is constrained. A second laser pulse (red shading), perpendicularly polarised and applied following the first, breaks the lab frame cylindrical symmetry. For molecules whose $\mathrm{O}$ axis is aligned due to the first pulse, this second laser field additionally exerts a torque about the $\mathrm{O}$ axis which "kicks" the second most polarizable axis towards alignment with the second laser polarization. For $\mathrm{SO}_{2}$, this second most polarizable axis (called hereafter the $\mathrm{S}$ axis) lies in the molecular plane along the $C_{2 v}$ axis (bisecting the $\mathrm{O}-\mathrm{S}-\mathrm{O}$ bond angle). The molecules now additionally proceed towards alignment around this new direction and FF3DA is produced after the second laser pulse, as shown in the third frame of Fig. 1. Some molecular $\mathrm{O}$ axes may not be well aligned at the time of application of the second laser pulse and will suffer a torque which misaligns their $\mathrm{O}$ axis. However, the flexibility of our method allows us to choose the duration, intensity, and time separation of the two laser fields so that we can optimize FF3DA of the ensemble, illustrated in the third frame of Fig. 1.

In order to unambiguously demonstrate FF3DA, we employ coincidence Coulomb explosion imaging which determines the complete 3D lab frame orientation of each molecule $[9,10]$. For each molecule measured, we determine two angles, $\vartheta_{\mathrm{O}}$ and $\vartheta_{\mathrm{S}}$, depicted in Fig. 2. These two angles (each being a function of the Euler angles) measure the alignment of the $\mathrm{O}$ and $\mathrm{S}$ axes respectively: $\vartheta_{\mathrm{O}}$ is the angle between the first laser pulse polarization direction (which defines the lab $x$ axis) and the projection of the $\mathrm{O}$ axis onto the lab $x y$ plane; $\vartheta_{\mathrm{S}}$ is the angle between the second laser polarization (which defines the lab $y$ axis) and the projection of the $\mathrm{S}$ axis on the $y z$ plane. For a

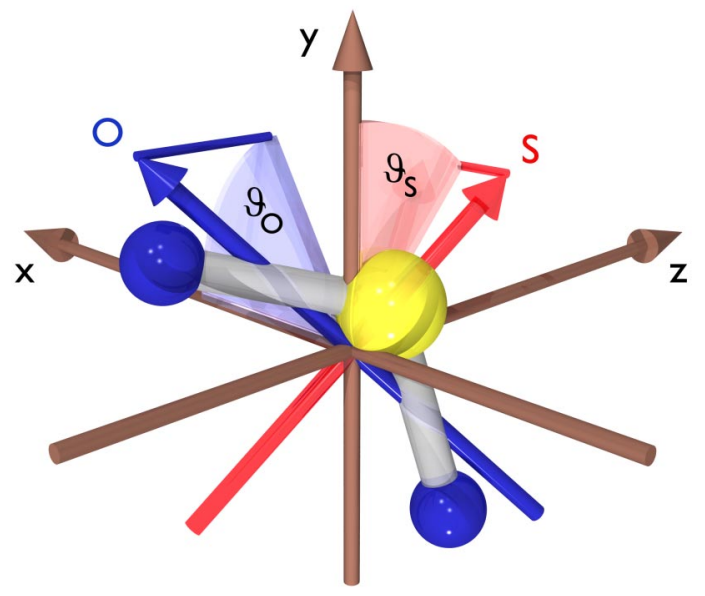

FIG. 2 (color online). Definition of angles $\vartheta_{\mathrm{O}}$ and $\vartheta_{\mathrm{S}}$. The laboratory frame is defined by the propagation direction of the laser pulses (the $z$ axis) and the polarization directions of the two linearly polarized alignment pulses; the first pulse is polarized along the $x$ axis, the second along the $y$ axis. perfectly 3D aligned (not oriented) molecule, these angles would simultaneously take values of either $0^{\circ}$ or $180^{\circ}$. We use the expectation values $\left\langle\cos ^{2} \vartheta_{\mathrm{O}}\right\rangle$ and $\left\langle\cos ^{2} \vartheta_{\mathrm{S}}\right\rangle$ to characterize the distribution of these angles for the ensemble of molecules. For each of these angles, which are measured in a plane, if the angle $\vartheta$ were uniformly distributed (i.e., no alignment) then $\left\langle\cos ^{2} \vartheta\right\rangle$ would take a value of 0.5 . This is in contrast to the value of $1 / 3$ for $\left\langle\cos ^{2} \theta\right\rangle$ when $\theta$ is a polar angle not confined to a plane. As an axis alignment increases, the associated expectation value $\left\langle\cos ^{2} \vartheta\right\rangle$ would increase towards 1 as $\vartheta$ becomes localized towards $0^{\circ}$ and $180^{\circ}$. Three-dimensional alignment is therefore characterized by $\left\langle\cos ^{2} \vartheta_{\mathrm{S}}\right\rangle>0.5$ and $\left\langle\cos ^{2} \vartheta_{\mathrm{O}}\right\rangle>0.5$ occurring simultaneously.

Our coincidence Coulomb explosion imaging measurements of FF3DA are shown in Fig. 3. A supersonic expansion of $0.5 \% \mathrm{SO}_{2}$ in argon was used to produce a rotational temperature of approximately $10 \mathrm{~K}$. The aligning pulses had an intensity of $\sim 2 \times 10^{13} \mathrm{~W} / \mathrm{cm}^{2}$ and were centered at a wavelength of $815 \mathrm{~nm}$. Optimal FF3DA was achieved with a pulse separation of $400 \mathrm{fs}$, while pulse separations of 200 fs and 600 fs show reduced FF3DA. Experimental data points are plotted along with curves obtained from classical simulations, showing good general agreement. Prior to the arrival of the alignment pulses, the ensemble is randomly aligned and both $\left\langle\cos ^{2} \vartheta\right\rangle$ have values of 0.5 . Application of the first aligning pulse increases $\left\langle\cos ^{2} \vartheta_{\mathrm{O}}\right\rangle$ towards 1 , as the $\mathrm{O}$ axis aligns to the first laser polarization. Note that $\left\langle\cos ^{2} \vartheta_{\mathrm{S}}\right\rangle$ remains 0.5 since the cylindrical symmetry of the interaction means that the $\mathrm{S}$ axis stays uniformly distributed about this laser polarization direction. As the alignment of the $\mathrm{O}$ axis continues to increase, a delayed, perpendicularly polarized laser pulse is applied to align the $\mathrm{S}$ axis to this second laser polarization, breaking the lab frame cylindrical symmetry. This produces maximal FF3DA at $\sim 500 \mathrm{fs}$ after this second laser pulse, as indicated by the dashed line. For $\mathrm{SO}_{2}$, rotation about the $\mathrm{O}$ axis has the lowest moment of inertia of the three axes, and therefore the second laser pulse has a shorter duration (50 fs FWHM) than the first (180 fs FWHM). For molecules not yet aligned to the $x$ axis, the second laser field tends to torque their $\mathrm{O}$ axis towards the $y$ polarization axis. Thus, the application of the second laser pulse can reduce the alignment of the $\mathrm{O}$ axis to the first laser polarization ( $x$ axis). Reducing the time delay between the two pulses to $200 \mathrm{fs}$ increases this deleterious effect, reducing the degree of FF3DA. Increasing the time delay to $600 \mathrm{fs}$, such that the second pulse arrives nearer to the maximal alignment of the $\mathrm{O}$ axis with the first pulse, results in a faster reduction of the alignment of the $\mathrm{O}$ axis and a reduced degree of FF3DA.

The Coulomb explosion probe was a third time-delayed femtosecond laser pulse of duration 50 fs copropagated with the aligning pulses. This pulse had sufficient intensity $\left(\sim 5 \times 10^{15} \mathrm{~W} / \mathrm{cm}^{2}\right)$ to multiply ionize a molecule. 

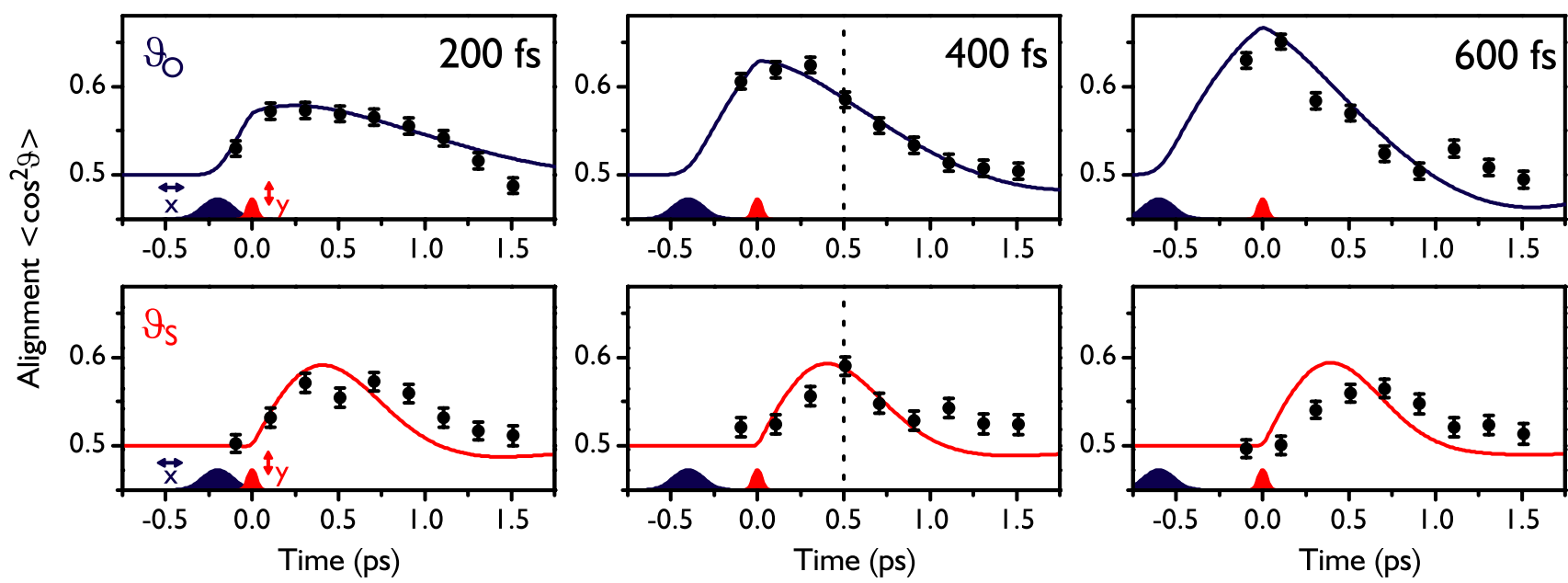

FIG. 3 (color online). Measured time dependence of $\mathrm{SO}_{2}$ molecular axis alignment. The three columns show data for pulse separations of $200 \mathrm{fs}$ (left), $400 \mathrm{fs}$ (middle), and $600 \mathrm{fs}$ (right). The zero of time is defined by the peak of the second laser pulse. Experimental data points are shown in black with error bars corresponding to the standard error of the mean. The solid lines are the results of classical calculations. The dashed line shows the maximum FF3DA achieved.

Following ionization, the molecule explodes due to the Coulomb repulsion between the ionized atoms. The 3D velocity vector of each fragment was measured using a time and position sensitive delay line anode detector in a time-of-flight spectrometer [9]. The detector system employed allowed for coincident detection of ion arrivals. By selecting coincident arrival events of two $\mathrm{O}$ ions and $1 \mathrm{~S}$ ion whose momenta sum to zero (and so originate from the same molecule) we are able to understand the characteristics of each explosion channel [10]. From these fully correlated events we were able to reconstruct the molecular geometry prior to explosion, which reproduces well the known bond angle of $\mathrm{SO}_{2}$. This fact allows us to unambiguously determine the $3 \mathrm{D}$ orientation of the molecule in the lab frame. Although the bond length measured by this method is roughly twice that of equilibrium $\mathrm{SO}_{2}$, indicative of enhanced ionization [10], this does not affect the measurement of the lab frame orientation.

To characterize the 3D alignment of a molecule, only two fragments are necessary. Since it is much more probable to detect two fragments rather than all three, we chose to look at the oxygen pair $\mathrm{O}^{2+}$ and $\mathrm{O}^{3+}$, with the unmeasured companion being mostly $\mathrm{S}^{2+}$ or $\mathrm{S}^{3+}$. Using our knowledge from fully correlated data, we verify that an $\mathrm{O}^{2+}$ and $\mathrm{O}^{3+}$ pair comes from the same molecule by filtering for a pair momentum sum between $40 \times 10^{-23}$ and $85 \times 10^{-23} \mathrm{~kg} \mathrm{~m} / \mathrm{s}$, an $\mathrm{O}^{2+}$ kinetic energy between 30 and $55 \mathrm{eV}$, and an $\mathrm{O}^{3+}$ kinetic energy between 40 and $75 \mathrm{eV}$. From the fully correlated three particle measurements, it was established that the oxygen velocities are roughly along the direction of the SO bonds, and so we find the direction of the $\mathrm{O}$ and $\mathrm{S}$ axes by taking the difference and sum, respectively, of the normalized oxygen velocity vectors.
The alignment of the $\mathrm{O}$ axis was measured with a pulse circularly polarized in the $x y$ plane (probe I) as in Fig. 2, and the alignment of the $\mathrm{S}$ axis was measured with a pulse linearly polarized along the $x$ axis (probe II). The symmetries of these probe geometries ensures that there can be no probe induced rotation in the plane of the measured angle [9]. For both measurements, probe-only Coulomb explosion data from a randomly oriented gas sample was used to characterize the nonuniform orientation dependence of the ionization efficiency, and any detector defects. The observed bias for ionization of molecules whose $\mathrm{O}$ axes are oriented towards the direction of probe polarization was included in the calculations shown in Fig. 3. The dependence of the ionization efficiency upon rotation of the molecule about the $\mathrm{O}$ axis for probe $\mathrm{I}$ (which in the case of very strong alignment of the $\mathrm{S}$ axis by the second alignment pulse, may artificially increase the measured $\mathrm{O}$ axis alignment) was not included in the calculations shown in Fig. 3, and was found to be a negligible effect as shown by the agreement between experiment and calculation. For probe II, the dependence of the ionization rotation about the $\mathrm{O}$ axis was found to be negligible. We collected about two thousand oxygen pairs for each time delay and each probe polarization.

Importantly, we note that this two pulse prompt alignment technique is quite resilient to temperature variation of the sample. We repeated these $\mathrm{SO}_{2}$ experiments without using argon carrier gas in the molecular beam. In this case, we expect that the rotational temperature of the $\mathrm{SO}_{2}$ molecules is $\gtrsim 100 \mathrm{~K}$. Nevertheless, this technique still produced measurable FF3DA, albeit reduced in value. This demonstrates the robustness of this approach. As our nonresonant technique relies only on the interaction with molecular polarizability, this approach is general. The 
pulse widths, separation, and intensities may be independently optimized for the rotational constants of the molecule, as well as the orientation of the polarizability ellipse with respect to the inertial ellipse. We note that the degree of FF3DA achieved here is comparable to that routinely achieved in 1D alignment experiments [4]. However, the alignment that we show is not fully optimized. Well developed optimal control technology [11] can be profitably applied to improve the degree of FF3DA and will be an area of future research. This technique is readily scalable to larger molecules, such as aromatic hydrocarbons, for which 1D field-free alignment was achieved [12]. To scale this technique to still larger molecules, it may be necessary to employ longer wavelength laser fields to avoid loworder resonances, ensuring that the Stark interaction with the molecular polarizability is dominant. With the addition of half-cycle terahertz fields [13], it may also be possible to produce field-free 3D orientation $[2,14]$.

It is important to note that even in this small molecule, the observed field-free 3D alignment shown in Fig. 3 persists for $\sim 300 \mathrm{fs}$. This is sufficiently long to permit a variety of structural or dynamical measurement techniques to be applied to FF3DA molecules. In fact, there is a major ongoing effort to develop approaches to probing molecular structures and dynamics using scattering or diffraction techniques, as opposed to spectroscopic methods. The development of "fourth generation" synchrotron light sources beckons the measurement of molecular structures via the diffraction of short $(<100 \mathrm{fs}) \mathrm{x}$-ray light pulses [15]. Complementary techniques include electron diffraction [16] laser-induced electron diffraction [17], high harmonic generation [18] and tomographic imaging [19], and multidimensional femtosecond coincidence spectroscopy [20]. These techniques, when combined with field-free 3D molecular axis alignment, will yield much more detailed information about molecular structure and dynamics.

K. F. L. acknowledges support from the Natural Sciences and Engineering Research Council of Canada (NSERC) and the Canadian Institute for Photonic Innovations (CIPI). J.G. U. acknowledges the support of the British CouncilNRC Researcher Exchange program and support through EPSRC Grant No. EP/C530756/1.

*Electronic address: j.underwood@open.ac.uk

[1] J. J. Larsen, K. Hald, N. Bjerre, H. Stapelfeldt, and T. Seideman, Phys. Rev. Lett. 85, 2470 (2000).

[2] H. Tanji, S. Minemoto, and H. Sakai, Phys. Rev. A 72, 063401 (2005).

[3] K. Yamanouchi, Science 295, 1659 (2002).

[4] H. Stapelfeldt and T. Seideman, Rev. Mod. Phys. 75, 543 (2003).

[5] C.Z. Bisgaard, M. D. Poulsen, E. Péronne, S. S. Viftrup, and H. Stapelfeldt, Phys. Rev. Lett. 92, 173004 (2004).
[6] J. G. Underwood, M. Spanner, M. Y. Ivanov, J. Mottershead, B. J. Sussman, and A. Stolow, Phys. Rev. Lett. 90, 223001 (2003).

[7] J.P. Heritage, T. K. Gustafson, and C. H. Lin, Phys. Rev. Lett. 34, 1299 (1975).

[8] J. G. Underwood, B. J. Sussman, and A. Stolow, Phys. Rev. Lett. 94, 143002 (2005).

[9] P. W. Dooley, I. V. Litvinyuk, K. F. Lee, D. M. Rayner, M. Spanner, D. M. Villeneuve, and P. B. Corkum, Phys. Rev. A 68, 023406 (2003).

[10] F. Légaré, K. F. Lee, I. V. Litvinyuk, P. W. Dooley, S. S. Wesolowski, P. R. Bunker, P. Dombi, F. Krausz, A. D. Bandrauk, and D. M. Villeneuve et al., Phys. Rev. A 71, 013415 (2005).

[11] Y. Silberberg, Nature (London) 430, 624 (2004); A. Assion, T. Baumert, M. Bergt, T. Brixner, B. Kiefer, V. Seyfried, M. Strehle, and G. Gerber, Science 282, 919 (1998); T. Brixner, G. Krampert, T. Pfeifer, R. Selle, G. G. M. Wollenhaupt, O. Graefe, C. Horn, and D. Liese et al., Phys. Rev. Lett. 92, 208301 (2004).

[12] E. Peronne, M. D. Poulsen, C. Z. Bisgaard, H. Stapelfeldt, and T. Seideman, Phys. Rev. Lett. 91, 043003 (2003).

[13] D. Daems, S. Guérin, D. Sugny, and H. R. Jauslin, Phys. Rev. Lett. 94, 153003 (2005); E. Gershnabel, I. S. Averbukh, and R. J. Gordon, Phys. Rev. A 73, 061401 (2006).

[14] H. Sakai, S. Minemoto, H. Nanjo, H. Tanji, and T. Suzuki, Phys. Rev. Lett. 90, 083001 (2003).

[15] J. Miao, K. O. Hodgson, and D. Sayre, Proc. Natl. Acad. Sci. U.S.A. 98, 6641 (2001); R. Neutze, R. Wouts, D. van der Spoel, E. Weckert, and J. Hajdu, Nature (London) 406, 752 (2000).

[16] J.C. H. Spence and R. B. Doak, Phys. Rev. Lett. 92, 198102 (2004); J.C. Williamson, J. Cao, H. Ihee, H. Frey, and A.H. Zewail, Nature (London) 386, 159 (1997); J. S. Baskin and A.H. Zewail, Chem. Phys. Chem. 6, 2261 (2005); R. Srinivasan, J. S. Feenstra, S. T. Park, S. Xu, and A. H. Zewail, Science 307, 558 (2005); D.W.H. Rankin and H.E. Robertson, Spectrosc. Prop. Inorg. Organomet. Compd. 37, 173 (2005); B. J. Siwick, J. R. Dwyer, R. E. Jordan, and R. J. D. Miller, Science 302, 1382 (2003); K. Hoshina, K. Yamanouchi, T. Ohshima, Y. Ose, and H. Todokoro, J. Chem. Phys. 118, 6211 (2003).

[17] H. Niikura, F. Légaré, R. Hasbani, A. D. Bandrauk, M. Y. Ivanov, D. M. Villeneuve, and P. B. Corkum, Nature (London) 417, 917 (2002); S. N. Yurchenko, S. Patchkovskii, I. V. Litvinyuk, P. B. Corkum, and G. L. Yudin, Phys. Rev. Lett. 93, 223003 (2004).

[18] S. Baker, J.S. Robinson, C. A. Haworth, H. Teng, R. A. Smith, C. C. Chirila, M. Lein, J. W. G. Tisch, and J.P. Marangos, Science 312, 424 (2006).

[19] J. Itatani, J. Levesque, D. Zeidler, H. Niikura, H. Pépin, J. C. Kieffer, P. B. Corkum, and D. M. Villeneuve, Nature (London) 432, 867 (2004).

[20] O. Gessner, A. M. D. Lee, J.P. Shaffer, H. Reisler, S. V. Levchenko, A. I. Krylov, J. G. Underwood, H. Shi, A. L. L. East, and E.t.H. Chrysostom et al., Science 311, 219 (2006). 